\title{
Accelerating newborn survival in Ghana through a low-dose, high-frequency health worker training approach: a cluster randomized trial
}

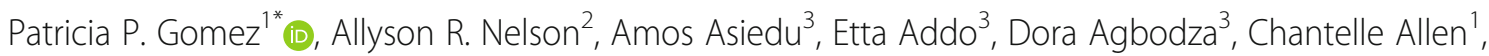
Martha Appiagyei ${ }^{3}$, Cynthia Bannerman ${ }^{4}$, Patience Darko ${ }^{3}$, Julia Duodu ${ }^{3}$, Fred Effah ${ }^{3}$ and Hannah Tappis ${ }^{1}$

\begin{abstract}
Background: Newborn deaths comprise nearly half of under-5 deaths in Ghana, despite the fact that skilled birth attendants (SBAs) are present at $68 \%$ of births, which implies that evidence-based care during labor, birth and the immediate postnatal period may be deficient. We assessed the effect of a low-dose, high-frequency (LDHF) training approach on long-term evidence-based skill retention among SBAs and impact on adverse birth outcomes.

Methods: From 2014 to 2017, we conducted a cluster-randomized trial in 40 hospitals in Ghana. Eligible hospitals were stratified by region and randomly assigned to one of four implementation waves. We assessed the relative risks (RRs) of institutional intrapartum stillbirths and 24-h newborn mortality in months 1-6 and 7-12 of implementation as compared to the historical control period, and in post-intervention facilities compared to pre-intervention facilities during the same period. All SBAs providing labor and delivery care were invited to enroll; their knowledge and skills were assessed pre- and post-training, and 1 year later.

Results: Adjusting for region and health facility type, the RR of 24-h newborn mortality in the 40 enrolled hospitals was $0.41(95 \% \mathrm{Cl} 0.32-0.51 ; p<0.001)$ in months $1-6$ and $0.30(95 \% \mathrm{Cl} 0.21-0.43 ; p<0.001)$ in months $7-12$ compared to baseline. The adjusted RR of intrapartum stillbirth was $0.64(95 \% \mathrm{Cl} 0.53-0.77 ; p<0.001)$ in months $1-6$ and $0.48(95 \% \mathrm{Cl} 0.36-0.63 ; p<0.001)$ in months $7-12$ compared to baseline. Four hundred three SBAs consented and enrolled. After 1 year, 200 SBAs assessed had 28\% (95\% Cl 25-32; $p<0.001)$ and 31\% (95\% Cl 27-36; $p<0.001)$ higher scores than baseline on low-dose 1 and 2 content skills, respectively.
\end{abstract}

Conclusions: This training approach results in a sustained decrease in facility-based newborn mortality and intrapartum stillbirths, and retained knowledge and skills among SBAs after a year. We recommend use of this approach for future maternal and newborn health in-service training and programs.

Trial registration: Retrospectively registered on 25 September 2017 at Clinical Trials, identifier NCT03290924.

Keywords: Pregnancy, Labor, Birth, Stillbirth, Newborn, Resuscitation, Training, Skills, Skilled birth attendant, Mentor

\footnotetext{
* Correspondence: patricia.gomez@jhpiego.org

'Jhpiego/Baltimore, 1615 Thames Street, Baltimore, MD 21232, USA

Full list of author information is available at the end of the article
} 


\section{Background}

The newborn mortality rate decreased by $47 \%$ worldwide between 1990 and 2015, from 36 to 19 deaths per 1000 live births, but the rate has declined more slowly in most low-resource settings, and is still far from the Sustainable Development Goal of less than 12 newborn deaths per 1000 live births by $2030 .^{1}$ Nearly half of newborn deaths take place during the first day of life [1]. Intrapartumrelated events are now the third leading cause of all deaths among children under age 5 (U5 mortality) [2]. Rates of intrapartum stillbirths are a measure of the quality of care during labor, and newborn mortality in the $24 \mathrm{~h}$ following birth can reflect the quality of care during labor as well as immediate postnatal care [3].

Ghana's U5 mortality rate is 60/1000 live births and the newborn mortality rate is $29 / 1000$ live births. Thus, newborn mortality represents $48 \%$ of U5 mortality despite the fact that $68 \%$ of births occur with a skilled birth attendant $(\mathrm{SBA})^{2}$ present [4]. The country's stillbirth rate is estimated at 22/1000 total births, although this estimate is not disaggregated by fetal deaths prior to labor or intrapartum stillbirths, and accurate information about the incidence of intrapartum stillbirth is lacking [5]. Two major causes of newborn deaths in Ghana, asphyxia (23\%) and sepsis (32\%), [6] could be prevented by increasing the use of evidence-based interventions during labor, birth, and the immediate postnatal period. The Ghana National Newborn Health Strategy and Action Plan 2014-2018 describes the country's goal to reduce institutional newborn mortality by at least $35 \%$ by 2018 through training of least $90 \%$ of SBAs in essential newborn care and basic resuscitation [6].

In-service training for SBAs is one of the most common interventions to address lack of knowledge and skills. However, these training interventions are seldom evaluated for effectiveness in improving learning or performance [7]. A literature review of effective in-service training described techniques that may improve learning outcomes and result in changes in performance [8]. The review found that didactic instruction often results in improved knowledge but no improvement in clinical practice. In contrast, the use of interactive techniques, including clinical simulation, case-based learning, hands-on practice with anatomic models, and immediate feedback on performance, results in greater improvement in knowledge and/or clinical practice than do passive techniques such as lecture or reading. Targeted, repeated training opportunities are preferable to one-time training and lead to the use of new skills in the clinical setting. Workplace learning may be superior for skill acquisition, and using multiple types of media can deliver training more efficiently. Thus, learning at the job site coupled with ongoing, repeated exposure to content is associated with greater improvement in clinical performance and health outcomes.
In collaboration with the Ghana Health Service (GHS), Jhpiego introduced interventions to reduce newborn mortality in 40 public and mission health facilities in which an ongoing quality improvement project ${ }^{3}$ was targeting reduction of U5 mortality. Using evidence from the literature review on in-service training, we designed and tested a low-dose, high-frequency (LDHF) training approach to update hospital-based SBAs in key evidence-based intrapartum and immediate newborn care practices, using current global guidelines [9] (Table 1).

All study sites received the full LDHF intervention that included two 4-day onsite sessions (low dose) with weekly practice sessions, SMS quizzes and reminders, and mentoring via mobile phone and onsite visits between trainings (high frequency). The low-dose sessions included competency acquisition through simulation, case-based learning, and small content packages spread over short time intervals (Table 2) [7, 10]. To foster a team-centered approach, [8] we trained all SBAs providing intrapartum and immediate postpartum care in each health facility, but limited each low-dose session to no more than ten SBAs. Thus, in large facilities more than one cycle of training was conducted to accommodate all eligible SBAs. This approach was considered low dose compared to the basic emergency obstetric and newborn care (BEmONC) training that was occurring in the country at the time of this study. The BEmONC training took place away from SBAs' usual workplace, included only a few SBAs from a given facility, and lasted up to 10 days.

We hypothesized that the LDHF approach to improving clinical skills would lead to a reduction in institutional intrapartum stillbirths and newborn mortality within $24 \mathrm{~h}$ of birth during the year of the intervention. We also hypothesized that the approach would result in improved and retained skills and knowledge among SBAs in maternity wards in study sites after a year.

Table 1 Components of low-dose, high-frequency approach. Lists the eight components that comprised the low-dose, high-frequency approach

\footnotetext{
- Two 4-day low-dose sessions (for facility skilled birth attendants)

- 1-day peer practice coordinator training after first low-dose session

- High-frequency practice sessions using MamaNatalie ${ }^{\circledR}$ and NeoNatalie ${ }^{\mathrm{TM}}$ anatomic models

- mMentoring with SMS reminder messages and quizzes

- Mentoring calls between master mentors and peer practice coordinators, and between project staff and master mentors

- Health information officer training

- Data collection and use training

- Supply of simulators, newborn resuscitation equipment, and delivery sets provided at first low-dose training session
} 
Table 2 Low-Dose Session Content. Lists the clinical content presented in each low-dose session

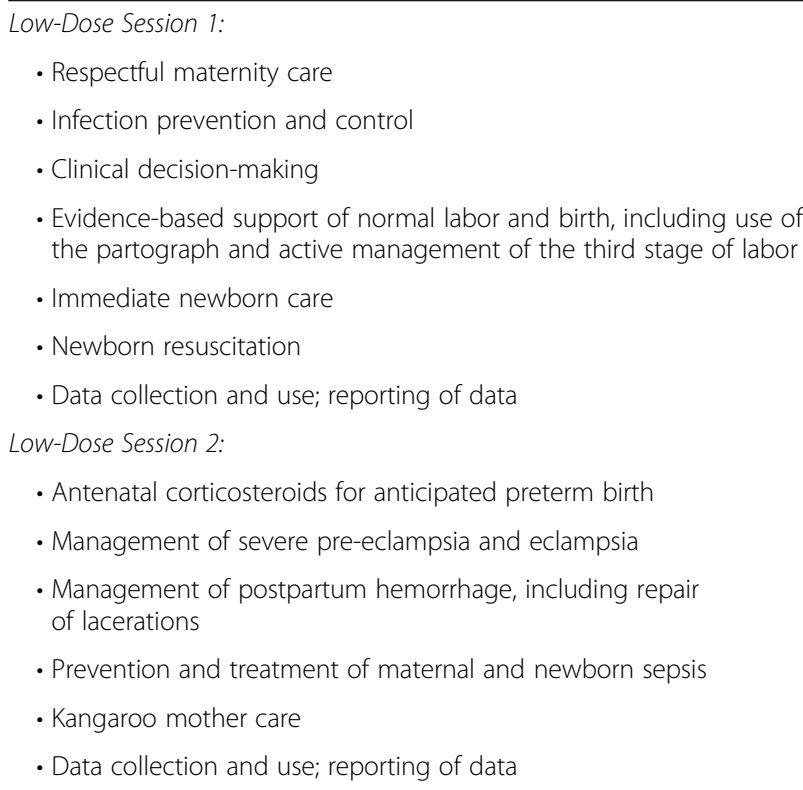

\section{Methods}

\section{Study design}

The study was a cluster-randomized trial implemented in 40 public and mission hospitals in Ghana between March 2014 and February 2017. Initial study sites included three regional hospitals, 38 district hospitals, and one polyclinic across Upper West, Central, and Western Regions of Ghana. Each facility was enrolled for 18 months. Data on institutional intrapartum stillbirths and 24-h newborn mortality were collected retrospectively for 6 months preintervention (i.e., baseline) and prospectively for 12 months post-intervention at each study site (Fig. 1).

All SBAs providing labor, birth, and immediate postnatal care at the selected facilities at the time of the first low-dose session were invited to participate. Four hundred and three SBAs individually consented and were enrolled in the study. All enrolled SBAs were registered or certified midwives.

\section{Randomization and masking}

Eligible facilities had at least three SBAs and an average of 30 or more births per month. These hospitals were stratified by region (three categories) and caseload (four categories), and then randomly assigned to one of four implementation waves. The pipeline randomization allowed for rigorous evaluation while the program was rolled out to all facilities. Waves 1 through 4 included eleven, seventeen, eight, and six facilities, respectively. Waves were unbalanced because of the stratification. Two hospitals were excluded at the time of implementation because they no longer met the inclusion criteria.
Neither facilities nor participants were masked due to the nature of the intervention.

\section{Procedures}

Experienced SBAs were prepared as regional master mentors (MMs) through a course in targeted BEmONC and training skills. MMs conducted the onsite, low-dose sessions and follow-up mentorship activities in collaboration with project staff. Of the 40 study facilities, 19 had a MM working within the facility, while the remaining facilities received mentoring support from MMs via mobile phone and periodic visits. During the first low-dose session at each site, two to three SBAs were trained as peer practice coordinators (PPCs) to lead the highfrequency practice sessions and were oriented to their roles in a day-long session. One month later, the second 4-day low-dose session was conducted. In the month between low-dose sessions and for 11 months afterward, SBAs carried out weekly PPC-led practice sessions with simulators and participated in mMentoring, which included mobile phone-based mentoring and onsite coaching with MMs and automated SMS reminders and quiz questions about key concepts presented during the low-dose sessions (Fig. 2).

MamaNatalie $^{\circledast}$ and NeoNatalie ${ }^{\mathrm{Ts}}$ simulators were given to each site to enable regular practice of targeted skills. Each site also received newborn resuscitation equipment (NeoNatalie resuscitator, penguin suction [Laerdal], and basic delivery sets). SBAs and health information officers collected service delivery data. Orientation to facilitylevel data collection was included in the first low-dose session for all SBAs. An additional training was provided to each facility's health information officer and maternity unit in-charge to ensure a common understanding of indicators and data collection, quality, and use. Monthly service statistics were extracted from routine health information systems and a supplementary delivery register introduced by the project. All outcome data were verified using register reviews at each study site.

Objective structured clinical examination (OSCE) tools and knowledge tests, based on global and national guidelines, were developed to assess SBAs in management of normal birth, immediate newborn care, and maternal and newborn complications. These assessments were used before and immediately after low-dose sessions, and again 1 year later, to evaluate knowledge and skill acquisition and retention. Master mentors and project staff conducted and collected all OSCE and knowledge test data. Assessments were scanned into Captricity (Oakland, CA, USA) for data capture and cleaning.

\section{Outcomes}

Twenty-four hour newborn mortality was defined as the death within $24 \mathrm{~h}$, or before discharge, of a newborn 


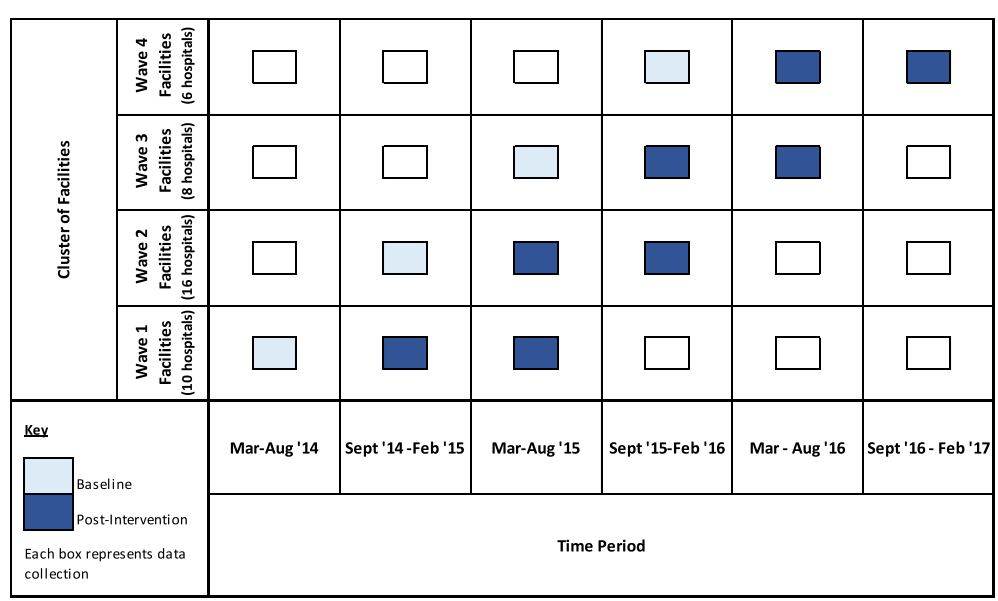

Fig. 1 Phased implementation and data collection. Describes timing of baseline and post-intervention data collection over the four waves of facility enrollment between March 2014 and February 2017

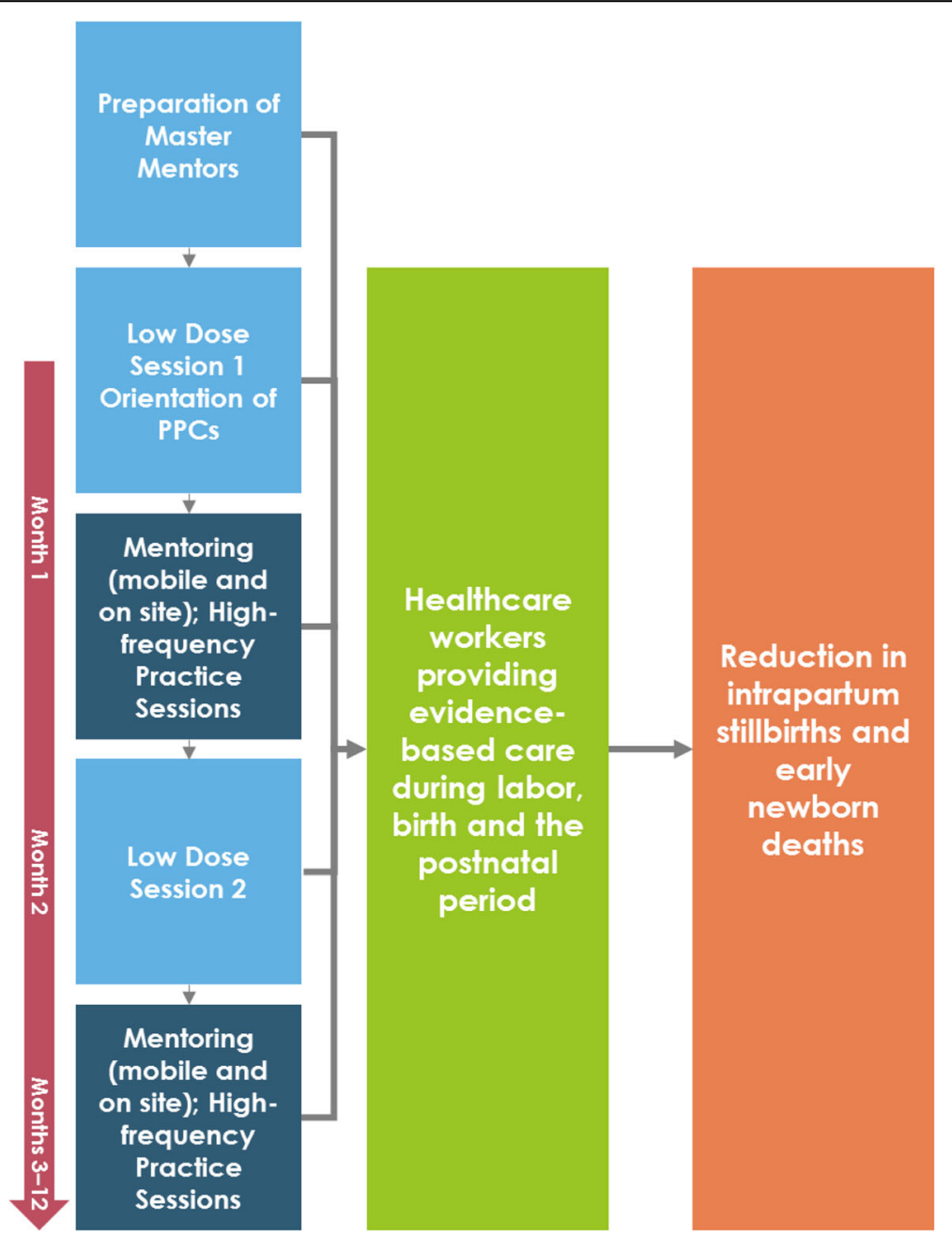

Fig. 2 Low-dose, high-frequency training approach to reduce newborn mortality and intrapartum stillbirths in Ghana. Describes the inputs, outcomes and impact of the project 
who breathed at birth. The institutional 24-h newborn mortality rate was defined as deaths in the first $24 \mathrm{~h}$ per 1000 facility live births. Intrapartum stillbirth was defined as the death of a baby who had heart tones present at the time of the mother's admission to the facility, was born with no signs of maceration, and did not breathe at birth, and on whom resuscitation attempts were unsuccessful. The institutional intrapartum stillbirth rate was defined as the proportion of all facility births that resulted in intrapartum stillbirth. Training results were the proportion of total test items (knowledge questions or skill steps) performed correctly.

\section{Statistical analysis}

We ran a simulation to assess statistical power to detect a reduction in the primary outcome, 24-h newborn mortality, in the original 42 study sites. In each simulation run, the study design was replicated, with four study entry time points (i.e., strata) for 42 facilities. A random effect Poisson model was fit to each study replicate to estimate the intervention effect on the number of newborn deaths, adjusting for the stratum, with the number of deliveries per facility as the offset term. Across the multiple iterations of the study, we calculated the empirical statistical power as the proportion of the simulation runs in which the $p$-value was less than 0.05 level of significance, indicating that the null hypothesis of no postintervention difference in the number of deaths is rejected. Results indicated that we had $99 \%$ power to detect a $25 \%$ decrease in the number of newborn deaths after the intervention.

We assessed institutional mortality rates and SBA competency at all facilities post-implementation compared to pre-implementation and assessed differences between institutional mortality rates at a subset of intervention and non-intervention (i.e., control) facilities during three 6-month time intervals. The entire study period (March 2014 to February 2017) was subdivided into four intervention waves, based on the start date of the intervention, and six calendar periods. For each facility the following variables were defined: intervention stage (coded as 0 for baseline, 1 for months 1-6 after Low-Dose 1, and 2 for months 7-12 after Low-Dose 1), wave (from 1 to 4 , depending on the start of the intervention), and calendar period (from 1 to 6). Exploratory analyses included comparison of the observed intrapartum stillbirth or newborn death rates by intervention stage, wave, calendar period, and region. Outcome rate trajectories were plotted over the calendar period by wave to assess for within-group trends. Baseline mortality rates were calculated as a facility-weighted (births or live births) average over the 6-month baseline period. Comparability of mortality rates at baseline was assessed using analysis of variance (ANOVA).
To estimate the effect of the intervention, we fit a series of generalized population-average linear models with negative binomial distribution and a log-link with exchangeable working correlation structure. The models were estimated using generalized estimating equations with robust variance estimates and a negative binomial distribution.

The base model included two indicator variables for intervention (months 1-6 and months 7-12) and adjustment for the region and level of the health facility (regional hospital or district hospital/polyclinic). Exponentiated beta coefficients for the two intervention variables were interpreted as the relative risk (RR) of the outcome post-intervention compared to the historical control (baseline) period.

To assess whether the effect of the intervention varied significantly by wave or region, the base model was modified to include the appropriate interaction terms. Secondary analyses were conducted using time as a continuous variable in a similar model to assess the monthly change in mortality rates prior to and after intervention. In addition, we used interaction terms in a modified model to assess the effect of the intervention comparing intervention and control facilities in the same calendar period, adjusting for effect of facility level on the outcome measures.

OSCE and knowledge test scores were calculated based on the number of steps correct in the OSCE or number of items correct on the knowledge test out of the total possible correct on each test. Scores were analyzed using ordinary least-squares linear regression that tested for a difference in individuals' pre- and post-test assessment scores, and pre-test and 1-year assessment scores, adjusting for clustering of SBAs within the facility.

Data were analyzed using Stata Version 14 (StataCorp LLC, College Station, TX, USA).

\section{Results}

In the 40 facilities, 403 SBAs consented and were enrolled in the study. Two hundred and one SBAs (50\%) were available for OSCE and knowledge assessment at 1 year after the intervention due to SBA rotation to other facilities, further education, and annual leave (Fig. 3).

The pre-intervention period included 38,192 births (Table 3). Months 1-6 and months $7-12$ included 36,160 and 31,498 births, respectively. The total numbers of 24-h newborn deaths and intrapartum stillbirths in the 40 sites, including baseline and the intervention periods, were 528 and 799, respectively.

Delivery caseloads, intrapartum stillbirth rates, and 24-h newborn mortality rates prior to intervention were comparable across waves (ANOVA $p=0.818$ and $p=0.527$, respectively) (Table 4 ). 


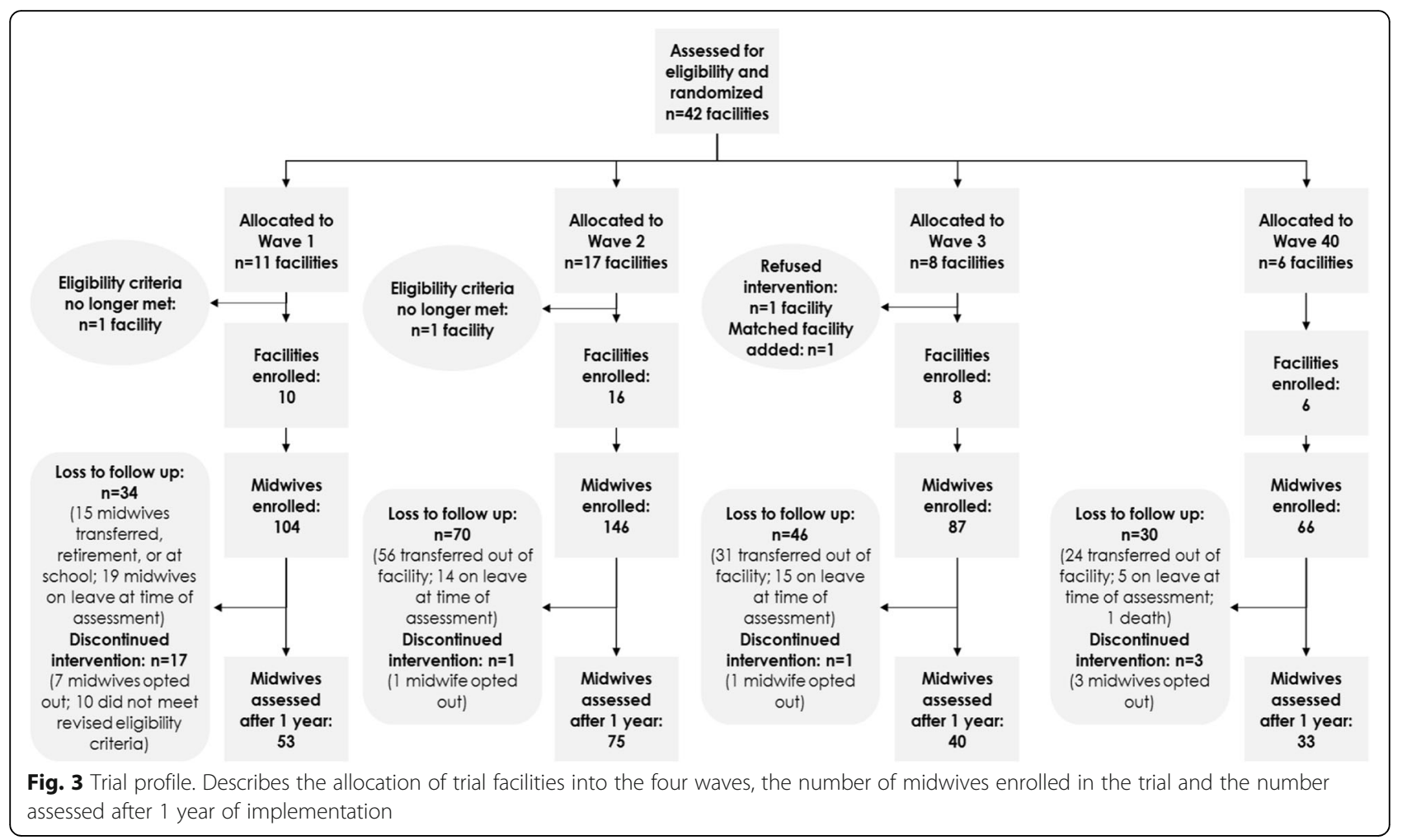

Implementation of the LDHF package resulted in a $55 \%$ reduction in risk of newborn mortality within $24 \mathrm{~h}$ of birth in the 6 months after LD1 (unadjusted RR 0.45, $95 \%$ CI $0.35-0.58 ; p<0.001)$ and a $65 \%$ reduction in risk in the next 6-month follow-up period (unadjusted RR 0.35 , 95\% CI $0.24-0.50 ; p<0.001$ ). After adjusting for region and facility level, the reduction in risk increased to $59 \%$ and $70 \%$, respectively (adjusted RRs $0.41,95 \%$ CI $0.32-0.51$ and $0.30,95 \%$ CI $0.21-0.43$, in months $1-6$ and months 7-12, respectively). Regional-level facilities had a significantly greater risk of 24-h newborn mortality compared to district-level facilities and polyclinics (RR 5.11, 95\% CI 3.55-7.36; $p<0 \cdot 001$ ).

The intervention resulted in a 35\% reduction (RR $0.65,95 \%$ CI $0.54-0.78 ; p<0001)$ in the risk of intrapartum stillbirth (unadjusted analysis) comparing months $1-6$ to the pre-intervention period, and a $51 \%$ reduction in risk (RR $0.49,95 \%$ CI $0.36-0.65 ; p<0.001$ ) in months 7-12 (Table 5). Results in a model adjusting for level of facility (regional vs. district level or polyclinic) and region did not substantially differ compared to the unadjusted model. Regional-level facilities had a significantly greater risk of intrapartum stillbirth compared to district-level facilities and polyclinics (RR 1.33, 95\% CI 1.03-1.70; $p=0.027$ ).

Pre-test scores on the low-dose session 1 (LD1) knowledge test and OSCE were $76 \%$ and $44 \%$, respectively (Table 6). Low-dose session 2 (LD2) pre-test scores were slightly higher at $91 \%$ and $52 \%$, respectively. Post-test scores increased by $11 \% \quad(95 \%$ confidence interval $[\mathrm{CI}] 9-12 ; p<0.001)$ and $4 \%(95 \%$ CI 2-6; $p=0.001)$ on LD1 and LD2 knowledge tests,

Table 3 Outcomes of interest during pre- and post-intervention periods

\begin{tabular}{llll}
\hline & Pre-intervention & Post-intervention \\
\cline { 2 - 4 } & Baseline & Months 1-6 & 36,160 \\
Number of births & 38,192 & 35,352 & 31,498 \\
Number of live births & 37,204 & 242 & 30,940 \\
Number of intrapartum stillbirths & 392 & 140 & 165 \\
Number of newborn deaths within 24 h of birth & 284 & 6.7 & 104 \\
Intrapartum stillbirth rate (per 1000 births) & 10.3 & 4.0 & 5.2 \\
24-h newborn mortality rate (per 1000 live births) & 7.6 & & 3.4 \\
\hline
\end{tabular}


Table 4 Baseline facility characteristics

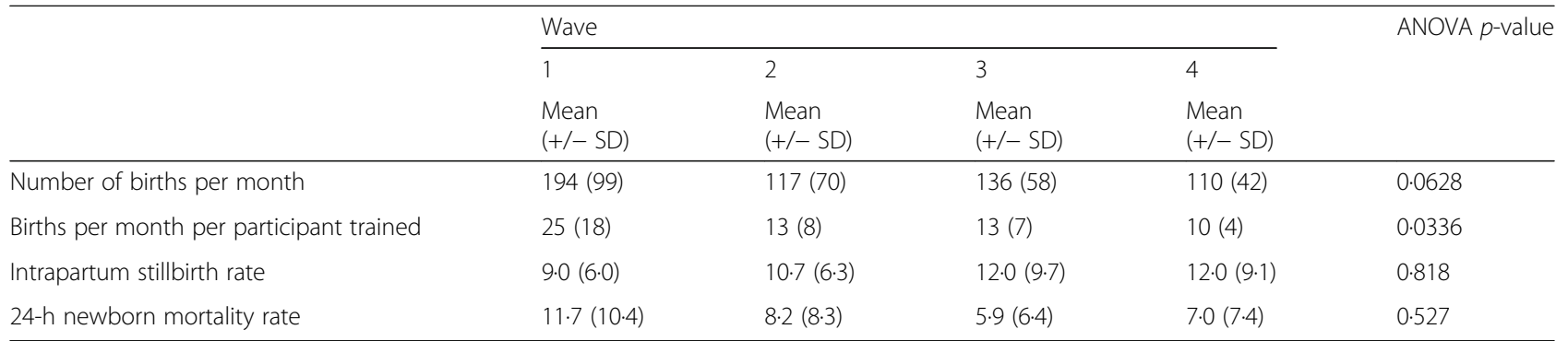

respectively, and $44 \%$ (95\% CI 40-48; $p<0.001)$ and $40 \%$ (95\% CI 37-43; $p<0.001)$ on the LD1 and LD2 OSCEs, respectively. After 1 year, participants retained most knowledge and skills gains. Participants scored 31\% and $28 \%$ higher on OSCE content from LD1 and LD2 at 1 year, respectively, compared to pre-test scores (LD1 95\% CI 27-36; $p<0.001$; LD2 95\% CI 25-32; $p<0.001)$, and $8 \%$ and $2 \%$ higher, respectively, on knowledge content from LD1 and LD2 at 1 year, compared to pre-test scores (LD1 95\% CI 6-10; $p<0.001 ;$ LD2 95\% CI 0-4; $p=0.025$ ).

There was no evidence of a temporal trend in intrapartum stillbirth or 24-h newborn mortality rates in study sites before the intervention. Therefore, the model did not adjust for calendar time. Once the intervention began, newborn mortality declined significantly from 1 month to the next over the one-year intervention period. Intrapartum stillbirth rates did not change significantly on a monthly basis; from this, we infer that the reduction in stillbirth rate was more drastic and immediate following the start of the intervention, and remained low for the remainder of the one-year follow-up period. Wave 3 facilities showed a greater reduction in risk of 24-h newborn mortality during months 7-12 postintervention compared to facilities in the other three waves. Risk of intrapartum stillbirth decreased more dramatically in wave 2 facilities compared to other facilities (Additional files 1 and 2: Tables S1 and S2). We found no significant difference in the mortality rates as a result of a master mentor being present at a study site.
We tested to see if there is a difference in the mortality rates between cohorts of facilities in the intervention phase and the baseline phase during the same calendar period. In all three time periods analyzed, newborn mortality rates were significantly lower in facilities in the intervention phase compared to those in the baseline phase; intrapartum stillbirth rates were also lower but only statistically significant in two of the three analyzed calendar periods. This analysis was conducted for a subset of the total study time and outcome observations, as the study was not designed or resourced to conduct a full randomization with comparison sites throughout the study duration.

\section{Discussion}

The primary aim of the study was to assess newborn outcomes. Results showed significant reduction in intrapartum stillbirth and newborn death within $24 \mathrm{~h}$. This could indicate that the quality of care during labor was sufficient to maintain maternal and fetal well-being. In addition, since resuscitation was attempted for all nonmacerated newborns not breathing at birth, many babies who would otherwise have been declared intrapartum stillbirths but actually had intrapartum-related asphyxia, were successfully resuscitated [11] corroborating the assertion by Wall et al. that training SBAs in newborn resuscitation could prevent up to $30 \%$ of mortality in full-term babies due to intrapartum-related events [12]. Reduction in newborn deaths within $24 \mathrm{~h}$ may be

Table 5 Mortality outcomes in 40 sites

\begin{tabular}{|c|c|c|c|c|c|c|}
\hline & Unadjusted risk ratio & $95 \% \mathrm{Cl}$ & $p$-value & Adjusted risk ratio ${ }^{a}$ & $95 \% \mathrm{Cl}$ & $p$-value \\
\hline $\begin{array}{l}\text { Pre-intervention } \\
\text { (6 months prior to intervention) }\end{array}$ & REF & REF & REF & REF & REF & REF \\
\hline \multicolumn{7}{|l|}{ Newborn death within $24 \mathrm{~h}$} \\
\hline Months 1-6 & 0.45 & $0.35-0.58$ & $<0.001$ & 0.41 & $0.32-0.51$ & $<0.001$ \\
\hline Months 7-12 & 0.35 & $0.24-0.50$ & $<0.001$ & 0.30 & $0.21-0.43$ & $<0.001$ \\
\hline \multicolumn{7}{|l|}{ Intrapartum stillbirth } \\
\hline Months 1-6 & 0.65 & $0.54-0.78$ & $<0.001$ & 0.64 & $0.53-0.77$ & $<0.001$ \\
\hline Months 7-12 & 0.49 & $0.36-0.65$ & $<0.001$ & 0.48 & $0.36-0.63$ & $<0.001$ \\
\hline
\end{tabular}

OSCE objective structured clinical examination

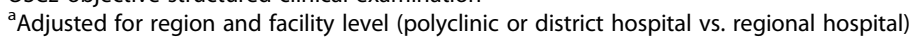


Table 6 Skilled birth attendant retention of key knowledge and skills

\begin{tabular}{|c|c|c|c|c|c|c|c|}
\hline & Pre-test (\%) & Post-test (\%) & $\begin{array}{l}\text { Difference (\%) } \\
\text { (post-/pre-) }\end{array}$ & $p$-value & $\begin{array}{l}\text { 1-year } \\
\text { assessment (\%) }\end{array}$ & $\begin{array}{l}\text { Difference (\%) } \\
\text { (1-yr/pre-) }\end{array}$ & $p$-value \\
\hline & Mean (+/- SD) & Mean (+/- SD) & Mean $(95 \% \mathrm{Cl})$ & & Mean (+/-SD) & Mean $(95 \% \mathrm{Cl})$ & \\
\hline \multicolumn{8}{|l|}{ Low-dose session 1} \\
\hline Knowledge assessment & $76(9)$ & $87(7)$ & $11(9-12)$ & $<0.001$ & $85(8)$ & $8(6-10)$ & $<0.001$ \\
\hline OSCE & $44(13)$ & $88(9)$ & $44(40-48)$ & $<0.001$ & $76(14)$ & $31(27-36)$ & $<0.001$ \\
\hline \multicolumn{8}{|l|}{ Low-dose session 2} \\
\hline Knowledge assessment & $91(10)$ & $95(6)$ & $4(2-6)$ & 0.001 & $94(10)$ & $2(0-4)$ & 0.025 \\
\hline OSCE & $52(14)$ & $92(7)$ & $40(37-43)$ & $<0.001$ & $81(12)$ & $28(25-32)$ & $<0.001$ \\
\hline
\end{tabular}

attributed to the use of newborn care interventions for every baby (immediate drying and warming, skin-toskin-contact, and immediate and exclusive breastfeeding) as well as rapid recognition and appropriate management of intrapartum-related asphyxia.

A second aim of the study was to assess the effect of the LDHF package on retention of key knowledge and skills after 1 year. We found that SBAs retained most knowledge and skills gained during low-dose sessions, which contributed to their ability to apply appropriate clinical interventions for improved newborn outcomes. The expectation after the low-dose sessions was that SBAs' skills should not be lost after training, but maintained or even improved. The lowest 1-year OSCE score occurred in relation to LD1, which dealt with care during normal labor and birth and newborn resuscitation. This implies that although SBAs attended many births, their practices did not reflect the evidence-based approaches presented during the first low-dose session. Thus, they might have needed more practice in simulated and clinical settings in the updated skills and/or reinforcement by PPCs or master mentors. Similarly, the skills for newborn resuscitation were new for most SBAs, and since resuscitation is a comparatively rare event, there may have been a need for reinforcement over time to maintain them. This is consistent with evaluations of maintenance of newborn resuscitation skills during which SBAs had the opportunity to review and practice skills on a regular basis after training [13].

In addition, our results demonstrate the impact and importance of an integrated package of evidence-based, clinical interventions that considers the continuum of care for the mother and baby on the day of birth. The components of the LDHF approach were chosen to target the major global causes of intrapartum-related events that are associated with up to $42 \%$ of maternal mortality, $32 \%$ of stillbirths, and $23 \%$ of newborn mortality [14]. They included cross-cutting elements of care that can improve maternal and newborn outcomes, such as respectful maternity care, which includes encouragement of continuous support during labor, ambulation, and positions of choice for labor and birth. These have been shown to decrease the duration of labor and the use of cesarean or assisted delivery $[15,16]$. Key evidencebased infection prevention practices were introduced during LD1 to decrease the risk of maternal and newborn sepsis. Hand hygiene, [17] instrument processing, [18] reduction of vaginal exams during labor, [19] avoiding routine rupture of membranes, [20] and prompt management of maternal fever and newborn sepsis were emphasized [21]. Severe pre-eclampsia and eclampsia are implicated globally in up to $4.7 \%$ of stillbirths, with the highest burden in south Asia and sub-Saharan Africa. Improved management of these disorders during labor could avert these deaths [22, 23]. Respectful maternity care and infection prevention were part of all OSCEs and skills checklists used by providers during training and high-frequency practice sessions.

The study emphasized correct use of the partograph to monitor labor and support clinical decision-making, which has been shown to improve identification of prolonged labor, a major contributor to perinatal mortality [24]. Best practices for immediate newborn care included immediate drying and skin-to-skin contact, [25, 26] delayed cord clamping, [27] and breastfeeding in the hour following birth [25].

Leading causes of maternal mortality in sub-Saharan Africa include hemorrhage (25\%), hypertension (16\%), and sepsis (10\%) [5]. Data for Ghana indicate that hemorrhage causes $24.3 \%$ of maternal mortality, hypertension accounts for $8.5 \%$, and sepsis is the cause of $6.7 \%$ of deaths [28]. Thus, the LDHF approach targeted management of severe pre-eclampsia/eclampsia, using magnesium sulfate and antihypertensive medications, [29] and prevention and treatment of postpartum hemorrhage [30].

One of the factors contributing to effective implementation of the LDHF approach was close collaboration with all levels of the Ghana Health Service. Nationallevel support was obtained at the outset, and regional health directors agreed to implementation in their regions. They also selected the MMs who carried out study activities as part of their normal duties; no MMs or SBAs were hired for the study. The investment in the 
development of a cadre of ten MMs in each region enabled close local follow-up of PPCs and SBAs in study facilities, both face-to-face and via mMentoring.

This study provides evidence that can guide strategies for health worker capacity-building at scale. Our results show a plausible causative association between use of an LDHF approach for facility-based in-service training and significant reductions in intrapartum stillbirths and newborn deaths in health facilities in low-resource settings. Health facilities did not undergo structural changes or receive additional equipment beyond delivery sets and resuscitation equipment. The LDHF approach built SBAs' competency in essential maternal and newborn health care practices. In each facility, all SBAs providing intrapartum and immediate postpartum care participated in this approach, resulting in facility-wide improvement in service quality, while minimizing the amount of time the SBAs spent outside of their facilities. It also enabled nearly all SBAs in each facility to receive the same training and follow-up support, which may have enhanced consistency of knowledge and skill transfer to the clinical setting. The critical mass of trained SBAs at each facility reinforces learning and builds a system of checks and balances throughout the health facility, likely rendering the change more sustainable. Coupled with ongoing practice and mentorship, improved practices are sustained and health outcomes continue to improve over time, even with staff attrition and turnover, as new SBAs are taught the skills by existing staff and can practice them on the simulators. Moreover, a pool of highly competent MMs enables continued application of the package after the study ends.

There are methodological limitations to consider in assessing the study implications. Because the study was embedded in program implementation, facility staff were aware of the outcome of interest, which could have affected their actions and performance. The presence of MMs at some facilities before intervention start-up could also have affected baseline outcome measurement rates. However, our analysis shows that mortality rates in all waves were comparable at baseline, and therefore we do not believe this has an effect on the results achieved. In addition, our study was not designed to be able to conduct a full time-series or randomized control trial analysis, due to resource limitations on baseline and control site data collection. Multiple analyses conducted using available data demonstrate qualitatively the same result. Other sources of potential bias include lack of concealment in group (i.e., wave) assignment and information bias such that data quality may have improved after the intervention started. In all likelihood, improved data quality following the intervention would result in more deaths being reported, rather than fewer, resulting in a smaller observed effect.
Although the regions for study implementation were chosen specifically to avoid overlap with other interventions conducted by the GHS and nongovernmental organizations, some SBAs from study facilities were invited to attend training on content related to BEmONC and/ or newborn resuscitation, but none of the training was similar to the LDHF approach. However, the influence of these activities on our outcomes cannot be ruled out.

Finally, while the technical approach demonstrates results, other factors such as cost and human resource availability (for trainers and mentors) must be considered. A nested costing study showed that the LDHF package was less expensive per participant compared to the traditional approach [31].

\section{Conclusions}

Implementation of an LDHF approach to train SBAs in key evidence-based intrapartum and immediate newborn care practices is associated with a significant reduction in the risks of intrapartum stillbirth and newborn mortality within $24 \mathrm{~h}$ of birth. The components of the approach were chosen specifically to address the most common causes of intrapartum stillbirth and early newborn death in Ghana and were reinforced in multiple ways: classroom learning and practice with simulators during and between low-dose sessions; peer-led practice and feedback between and after low-dose sessions; follow-up via targeted SMS messaging and support by a master mentor; and robust data collection and reporting. In addition, all training was done onsite and involved nearly all maternity ward SBAs at each site. These elements contributed to transfer of knowledge and skills to the clinical setting and thus to improved outcomes. The adoption of the LDHF approach in Ghana and other low-resource countries has the potential to contribute to the acceleration of newborn survival and the achievement of Sustainable Development Goal 3 (reduction of neonatal mortality to at least as low as 12 per 1000 by 2030).

Policymakers and health partners should review current approaches to improving service delivery in maternal and newborn health, and consider investing in and adopting this approach as an innovative and effective method of training health care workers. While further research is necessary, we believe this approach should be considered for the improvement of clinical services in other technical areas that mandate acquisition and retention of skills to improve health outcomes.

\section{Endnotes}

${ }^{1}$ UN Sustainable Development Goals (http://www.un. org/sustainabledevelopment/health/page/2/0; accessed 26 January 2017)

${ }^{2} \mathrm{~A}$ skilled attendant is an accredited health professional such as a midwife, doctor, or nurse who has been 
educated and trained to proficiency in the skills needed to manage normal (uncomplicated) pregnancies, childbirth, and the immediate postnatal period, and in the identification, management, and referral of complications in women and newborns. (WHO, ICM and FIGO. Making pregnancy safer: the critical role of the skilled attendant. A joint statement by WHO, ICM and FIGO. Geneva, 2004.)

${ }^{3}$ Project Fives Alive! is implemented by the Institute for Healthcare Improvement and the National Catholic Health Service in collaboration with the Ghana Health Service.

\section{Additional files}

Additional file 1: Table S1. Newborn mortality risk ratio by wave. Describes the effect of the intervention on newborn mortality by intervention wave. (DOCX $15 \mathrm{~kb}$ )

Additional file : Table S2. Intrapartum stillbirth risk ratio by wave. Describes the effect of the intervention on intrapartum stillbirth by intervention wave. (DOCX $15 \mathrm{~kb})$

\section{Abbreviations}

ANOVA: Analysis of variance; BEmONC: Basic emergency obstetric and newborn care; Cl: Confidence interval; GHS: Ghana Health Service; LD1: Low dose session 1; LD2: Low dose session 2; LDHF: Low dose, high frequency; MM: Master mentor; OSCE: Objective structured clinical examination; PPC: Peer practice coordinator; RR: Relative risk; SBA: Skilled birth attendant; SMS: Short message service; U5: Under 5

\section{Acknowledgements}

The authors thank The Bill \& Melinda Gates Foundation for their support of this work. They also thank the Institute for Healthcare Improvement and the director of its Project Fives Alive! initiative in Ghana, Dr. Sodzi Sodzi-Tetteh, for their fruitful collaboration during this study. We also extend great appreciation to the Ghana Health Service and the National Catholic Health Service for allowing us to carry out our work in their facilities. We especially recognize and are grateful to Mary Drake, Jhpiego Tanzania, for her contribution to the initial design of this study, and Gayane Yenokyan, Johns Hopkins Bloomberg School of Public Health, for her support in data analysis. We are indebted to Heather Harrison, Jhpiego Baltimore, who provided unfaltering program support and guidance throughout the study. The entire Ghana-based study team's rigorous fieldwork was inspiring and we congratulate them. Not least, we would like to thank the midwives and doctors who so enthusiastically participated in this study and made their constant priority the improvement of maternal and newborn care in their workplaces.

\section{Funding}

This research was funded by the Bill \& Melinda Gates Foundation. The funders had no role in data collection, data analysis, data interpretation, or manuscript development. The contents of this publication are the responsibility of the authors and do not necessarily reflect the views of the funder.

\section{Availability of data and materials}

The datasets from this study are available from the corresponding author on reasonable request.

\section{Authors' contributions}

$P G, M A$ and $C A$ contributed to the study design and $A A, E A, D A, M A, C A, P D$ $J D, F E$, and $A N$ to study implementation and data collection. AN, AA and HT performed data analysis. All authors (PG, AN, AA, EA, DA, CA, MA, CB, PD, JD, $\mathrm{FE}, \mathrm{HT}$ contributed to interpretation of findings, drafted the manuscript and contributed to manuscript revision. All authors read and approved the final manuscript.

\section{Authors' information}

N/A

\section{Ethics approval and consent to participate}

Study team members obtained written informed consent from all providers who participated in the study. Ethical approval and oversight were provided by Johns Hopkins Bloomberg School of Public Health Institutional Review Board (IRB\# 5442), Baltimore, MD, USA, and the Ghana Health Service Ethics Review Committee (ERC\# GHS-ERC-10/11/13), Accra, Ghana. This study did not involve the use of animals.

\section{Consent for publication}

Not applicable

\section{Competing interests}

The authors declare that they have no competing interests.

\section{Publisher's Note}

Springer Nature remains neutral with regard to jurisdictional claims in published maps and institutional affiliations.

\section{Author details}

'Jhpiego/Baltimore, 1615 Thames Street, Baltimore, MD 21232, USA. 2Jhpiego/Liberia, UN Drive, OPP Rock Compound, Mamba Point, Monrovia, Liberia. ${ }^{3}$ Jhpiego Ghana, 14 Ollenu Street, East Legon, Accra, Ghana. ${ }^{4}$ Ghana Health Service, Private Mail Bag, Ministries, Accra, Ghana.

Received: 10 October 2017 Accepted: 15 March 2018

Published online: 22 March 2018

\section{References}

1. World Health Organization. Children: reducing mortality. WHO fact sheet, updated September 2016. http://www.who.int/mediacentre/factsheets/fs178/en/ Accessed 23 Jan 2017.

2. Liu L, Oza S, Hogan D, et al. Global, regional, and national causes of under-5 mortality in 2000-15: an updated systematic analysis with implications for the sustainable development goals. Lancet. 2016;388:3027-35.

3. Lawn J, Blencowe H, Waiswa P, et al. Stillbirths: rates, risk factors, and acceleration towards 2030. Lancet. 2016;387:587-603.

4. Ghana Statistical Service (GSS), Ghana Health Service (GHS), and ICF International. Ghana demographic and health survey 2014. Rockville: GSS, GHS, and ICF International; 2015.

5. UNICEF and the World Health Organization. A decade of tracking progress for maternal, newborn and child survival: the 2015 report. Geneva: UNICEF and WHO; 2015.

6. Ghana Ministry of Health. Ghana national newborn health strategy and action plan 2014-2018. Accra: Ghana Ministry of Health; 2014.

7. Ameh CA, van den Broek N. Making it happen: training health-care providers in emergency obstetric and newborn care. Best Pract Res Clin Obstet Gynaecol. 2015;29(215):1077-91.

8. Bluestone J, Johnson P, Fullerton J, Carr C, Alderman J, Bon Tempo J. Effective in-service training design and delivery: evidence from an integrative literature review. Hum Resour Health. 2013;11(1):Article 51.

9. Sutton RM, Niles D, Meaney PA, et al. Low-dose, high-frequency CPR training improves skill retention of in-hospital paediatric providers. Paediatr. 2011;128(1):e145-51.

10. Anderson JM, Warren JB. Using simulation to enhance the acquisition and retention of clinical skills in neonatology. Semin Perinatol. 2011:35:59-67.

11. Goldenberg RL, McClure EM, Jobe AH, Kamath-Rayne BD, Gravette MG, Rubens CE. Stillbirths and neonatal mortality as outcomes. Int I Gynecol Obstet. 2013;123:252-3.

12. Wall SN, Lee ACC, Niermeyer $\mathrm{S}$, et al. Neonatal resuscitation in low-resource settings: what, who, and how to overcome challenges to scale up? Int J Gynecol Obstet. 2009;107:S47-64.

13. Ashish KC, Wrammert J, Nelin V, et al. Evaluation of Helping Babies Breathe Quality Improvement Cycle (HBB-QIC) on retention of neonatal resuscitation skills six months after training in Nepal. BMC Pediatr. 2017;17:103.

14. Lawn JE, Kinney M, Lee AC, et al. Reducing intrapartum-related deaths and disability: can the health system deliver? Int J Gynecol Obstet. 2009; 107(Suppl 1):S123-40. 
15. Lawrence A, Lewis L, Hofmeyr GJ, Dowswell T, Styles C. Maternal positions and mobility during first stage labor (Review). Cochrane Database Syst Rev. 2013;(8). https://doi.org/10.1002/14651858.CD003934.pub3.

16. Hodnett ED, Gates S, Hofmeyr GJ, Sakala C, Weston J. Continuous support for women during childbirth (Review). Cochrane Database Syst Rev. 2017;(7). https://doi.org/10.1002/14651858.CD003766.pub6

17. World Health Organization. WHO guidelines on hand hygiene in health care. Geneva: WHO; 2009.

18. World Health Organization (WHO) and Pan American Health Organization (PAHO). Decontamination and reprocessing of medical devices for healthcare facilities. Geneva: WHO and PAHO; 2016.

19. Downe S, Gyte GM, Dahlen HG, Singata M. Routine vaginal examinations for assessing progress of labour to improve outcomes for women and babies at term (Review). Cochrane Database Syst Rev. 2013;7:CD010088.

20. Smyth RMD, Markham C, Dowswell T. Amniotomy for shortening spontaneous labor (Review). Cochrane Database Syst Rev. 2013;(6). https:// doi.org/10.1002/14651858.CD006167.pub4.

21. World Health Organization. WHO recommendations for prevention and treatment of maternal peripartum infections. Geneva: WHO; 2015.

22. World Health Organization. WHO recommendations for management of common childhood conditions: evidence for technical update of pocket book recommendations. Geneva: WHO; 2012.

23. von Dadelszen P, Firoz T, Donnay F, et al. Preeclampsia in low and middle income countries-health services lessons learned from the PRE-EMPT (PREeclampsia-eclampsia monitoring, prevention \& treatment) project. J Obstet Gynaecol Can. 2012;34(10):917-26.

24. Hofmeyr GJ, Haws RA, Bergström S, et al. Obstetric care in low-resource settings: what, who, and how to overcome challenges to scale up? Int J Gynecol Obstet. 2009;107:S21-45.

25. American Academy of Pediatrics (AAP). Helping babies breathe provider guide. 2nd ed. AAP: Elk Grove Village; 2016.

26. Moore ER, Bergman N, Anderson GC, Medley N. Early skin-to-skin contact for mothers and their healthy newborn infants (Review). Cochrane Database Syst Rev. 2016;(11). https://doi.org/10.1002/14651858.CD003519.pub4.

27. World Health Organization. Guideline: delayed umbilical cord clamping for improved maternal and infant health and nutrition outcomes. Geneva: WHO; 2014.

28. Ghana Statistical Service (GSS), Ghana Health Service (GHS), and Macro International. Ghana maternal health survey 2007. Calverton: GSS, GHS, and Macro International; 2009.

29. World Health Organization. WHO recommendations for prevention and treatment of pre-eclampsia and eclampsia. Geneva: WHO; 2011.

30. World Health Organization. WHO recommendations for the prevention and treatment of postpartum haemorrhage. Geneva: WHO; 2012.

31. Willcox M, Harrison H, Asiedu A, Nelson A, Gomez P, LeFevre A. Incremental cost and cost-effectiveness of low-dose, high-frequency training in basic emergency obstetric and newborn care as compared to status quo: part of a cluster randomized training intervention evaluation in Ghana. Glob Health. 2017;13:88. https://doi.org/10.1186/s12992-017-0313-x. Published online 6 Dec 2017

\section{Submit your next manuscript to BioMed Central and we will help you at every step:}

- We accept pre-submission inquiries

- Our selector tool helps you to find the most relevant journal

- We provide round the clock customer support

- Convenient online submission

- Thorough peer review

- Inclusion in PubMed and all major indexing services

- Maximum visibility for your research

Submit your manuscript at www.biomedcentral.com/submit 